\title{
How accurately can spherical caps be represented by rational quadratic polynomials?
}

\author{
CHRISTOPHER G. PROVATIDIS \\ School of Mechanical Engineering \\ National Technical University of Athens \\ 9 Iroon Polytechniou, 15780 Zografou \\ GREECE
}

\begin{abstract}
This paper discusses the incapability of a tensor product rational quadratic patch to accurately represent a spherical cap. It was analytically found that there is no combination of control points and associated weights to accurately represent the spherical cap. On top of that, an optimization technique has revealed that for a unit sphere the computed radii in the parametric space can be limited to the interval [0.999999994, 1.000104146], which is practically a good approximation. This study makes sense as a preparatory stage in relation with the isogeometric analysis (IGA), which may be applied in conjunction with either the Finite Element Method (FEM) or the Boundary Element Method (BEM).
\end{abstract}

Key-Words: - Systems Theory, Dynamical Systems, Distributed Systems, Computational geometry, Spherical cap, Bernstein polynomials, Rational interpolation, Weights, Control points, Isogeometric analysis, FEM, BEM.

Received: December 28, 2020. Revised: May 31, 2021. Accepted: June 17, 2021. Published: June 28, 2021.

\section{Introduction}

Computer-aided geometric design (CAGD) deals with mathematical and computational methods for the description of geometric objects as they arise in areas ranging from $\mathrm{CAD} / \mathrm{CAM}$ to robotics and scientific visualization [1]. On the other point of view, Computer Methods such as the finite element method (FEM) start with the aforementioned object and operate sequentially by fulfilling the dominating physical laws at a computational mesh which is generated afterwards within this geometric object [2].

Unlike the two above discrete procedures, since October 2005, the so-called 'isogeometric analysis' (IGA) method has been proposed, which combines computational mechanics (FEM) and geometric model (CAGD) [3] thus bridging the gap between them. In more detail, IGA operates directly on the NURBS-based geometric model utilizing the involved set of weighted B-spline basis there and the associated control points. For an overview the interested reader may consult [4]. For details regarding the computer implementation as well as the differences between IGA with the classical FEM, the reader is referred to $[5,25]$. The advantage of IGA is that it can deal with exact representations of the involved geometries, particularly when conical sections appear. Therefore, circles, ellipses, spheres and ellipsoids can be ideally treated by IGA. Also, since a rather small number of parameters are involved in the IGA model, the shape optimization process is drastically facilitated.

Spheres and spherical parts, are frequently met in structural engineering (e.g., thin shells) and mechanical engineering (mostly rigid objects, e.g. ball joints etc.). In addition, spherical caps are of great interest in many scientific areas such as computer graphics [6], optics [7], solar engineering [8], geosciences [9] and fluid mechanics (oil pipelines, nuclear reactors) [10], among others. In all these cases, an accurate computational mechanics model is required in which the accurate representation of the geometry should be ensured. For example, it has been reported that buckling analysis is particularly sensitive when the spherical geometry is not well represented [11,12], and some of the latest studies on spherical caps are $[13,14]$.

Within this context, this paper restricts to the parameterization of spherical caps using a tensor product of nine rational basis functions, which are weighted quadratic Bernstein polynomials.

It is well known that an entire sphere can be treated as a solid by the revolution of a circular arc, of central angle equal to 180 degrees, about an axis. Also, half a sphere (such as its northern or southern part) may be easily produced by the rotation of a circular arc of central angle equal to 90 degrees about the vertical axis. In this particular case we may use three rational Bernstein-Bézier polynomials associated to three control points as follows. Two of 
them are taken at the ends of the circular arc and the third one at the intersection of the two tangents of the arc on an axial section at the aforementioned ends [15].

If however we wish to represent the four-sided (say) northern cap which is formed by an inscribed cube concentric with the sphere, then there are no guidelines about the minimum polynomial degree that has to be applied. One reported case is to use biquartic polynomials $[16,17]$, while the MATLAB command rsmak ('southcap') provides a 3vector valued rational of order five polynomial whose values on the unit square $[-1,1] \times[-1,1]$ fill out the aforementioned piece of the unit sphere [18]. A study on triangular and quadrangular quadratic Bézier patches to represent a quadric has been reported [19]. The latter has been followed by further studies [20-22] but the problem still remains open.

This paper goes beyond the state-of-the-art and mathematically proves the incapability of biquadratic rational Bézier interpolation to accurately represent quadrilateral spherical patches such as the abovementioned southern or northern cap. Not only that but is also presents an acceptable practical solution of high precision.

The paper is structured as follows. In section 2 we present the basic equations for the parametric representation of the spherical cap, which is considered as a generalized quadrilateral patch of CAGD type. In section 3 we present the conditions which have to be fulfilled to ensure that the computed points belong to a sphere of unit radius. In section 4 we present an optimization procedure to determine a good but not accurate approximation of the spherical cap. Section 5 is a discussion on the findings and the future research whereas section 6 is the conclusions. The paper is followed by an Appendix regarding the position of the control points along the four edges of the patch.

\section{Problem Formulation}

In this section we define the spherical cap in terms of geometry (subsection 2.1), in full detail. In subsection 2.2 we determine the control points and the corresponding weights along the four edges (boundary) of the quadrilateral patch. Finally, in subsection 2.3 we present the parametric equations of the interior of the spherical cap.

\subsection{Geometric details}

We restrict our analysis to a spherical patch which is produced on a unit sphere $(R=1)$ by an inscribed concentric cube.

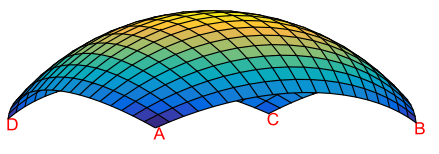

Fig. 1: Spherical cap

It is easy to validate that the length $a$ of each cube's side is given in terms of the radius $R$ by (see, Appendix A):

$$
a=\frac{2 R}{\sqrt{3}}
$$

It is clarified that if the upper (northern) face of the inscribed cube is denoted by $A B C D$, each curvilinear edge such as $A B$ of the spherical cap $A B C D$ is produced by connecting the side $A B$ of the cube with the centre $O$ of the sphere and then intersecting the plane $O A B$ with the surface of the sphere, and so on. The finally produced spherical cap is shown in Fig. 1.

Obviously, the abovementioned geometrical construction associated to the top face $A B C D$ of the cube defines exactly one-sixth of the sphere, thus the rest five faces of the cube define the rest five sixths of the same sphere's surface.

\subsection{Representation of patch edges}

In this subsection we determine the position and the weights of the control points along the four edges of the quadrilateral patch which approximates the spherical cap.

By construction, each side of the spherical cap is a perfect circular arc of a great circle of radius $R$, thus it can be accurately represented by three rational Bézier polynomials associated to a set of three control points $[15,23]$. These points are found at the corner points ( $A$ and $B$ for the edge $\mathrm{AB}$ ) as well as at the intersection of the tangents through $A$ and $B$ of the circular arc $A B$ on the plane $O A B$. Regarding the corresponding weights, one possibility is to use unit values at the ends. In this case, since the cosine of the angle formed by the chord $A B$ and the tangent at the end $A$ is $\cos \alpha=\sqrt{2 / 3}$ (for details, see Appendix A), the triplet of the weights will be:

$$
w_{0}=1, \quad w_{1}=\sqrt{\frac{2}{3}}, \quad w_{2}=1
$$


In the case that the Cartesian reference system Oxyz is chosen so as the vertical plane $O y z$ halves the inscribed cube (and the sphere as well) passing through the middle points of the edges $A B$ and $C D$ and being perpendicular to them, obviously the corner points $A$ and $B$ possess the following Cartesian coordinates:

$$
A\left(-\frac{a}{2},-\frac{a}{2}, \frac{a}{2}\right), \quad B\left(\frac{a}{2},-\frac{a}{2}, \frac{a}{2}\right),
$$

Then, following the above-mentioned construction using the tangents, the three control points corresponding to the circular arc $A B$ will be found at:

$$
\begin{aligned}
& P_{00}\left(-\frac{a}{2},-\frac{a}{2}, \frac{a}{2}\right) \quad \text { with } \quad w_{00}=1 \\
& P_{01}\left(0,-\frac{3 a}{4}, \frac{3 a}{4}\right) \text { with } \quad w_{01}=\sqrt{\frac{2}{3}} \\
& P_{02}\left(-\frac{a}{2},-\frac{a}{2}, \frac{a}{2}\right) \quad \text { with } \quad w_{00}=1
\end{aligned}
$$

Similar expressions are valid for the rest three circular edges, i.e. $B C, C D$ and $D A$, which are obtained by merely rotating the arc $A B$ in the anticlockwise direction by 90 degrees about the vertical axis of symmetry ( $z$-axis).

\subsection{Representation of the entire patch}

Following the standard computer-aided geometric design (CAGD) approach, the coordinates $\mathbf{x}(\xi, \eta)=[x, y, z]^{T}$ of any point $P(x, y, z)$ on the above-mentioned spherical cap is given in terms of the parameters $(\xi, \eta)$ of the patch by (see, [23]):

$$
\mathbf{x}(\xi, \eta)=\sum_{i=0}^{2} \sum_{j=0}^{2} R_{i j}(\xi, \eta) \mathbf{x}_{P_{i j}},
$$

where $\mathbf{x}_{P_{i j}}$ is the coordinate of the control point $P_{i j}$, and $R_{i j}$ is the rational basis functions which may be expressed in terms of the Bernstein polynomials $B_{i j}$ and the weights $w_{i j}$, as follows:

$$
R_{i j}(\xi, \eta)=\frac{w_{i j} B_{i}(\xi) B_{j}(\eta) \mathbf{x}_{P_{i j}}}{\sum_{i=0}^{2} \sum_{j=0}^{2} w_{i j} B_{i}(\xi) B_{j}(\eta)}
$$

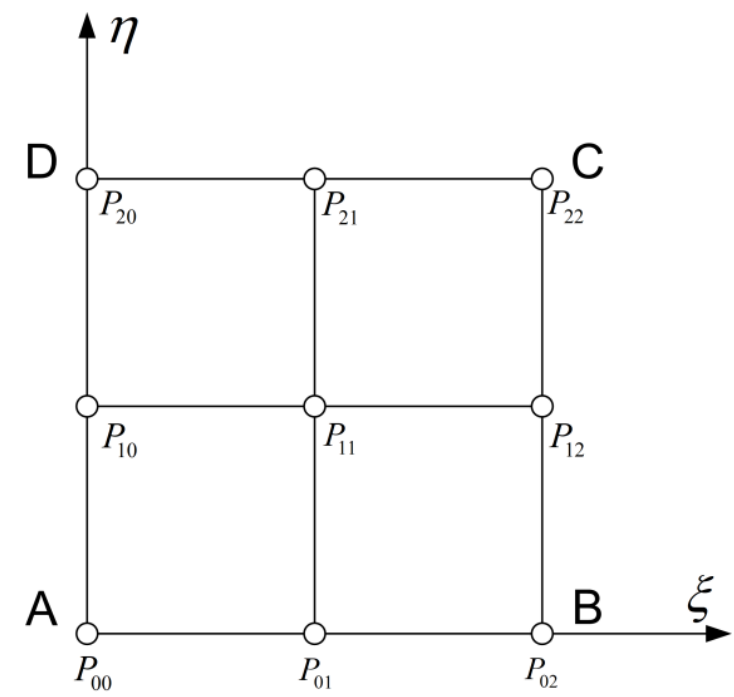

Fig. 2: Control points of the spherical patch, in the reference unit square.

Up to this point, we have determined the eight control points along the four curvilinear arcs (i.e., $A B, B C, C D$ and $D A$ ) and we seek for the central control point $P_{11}$ that is shown in Fig. 2.

We recall that the triplet of the quadratic Bernstein polynomials is given by:

$$
B_{0}(s)=(1-s)^{2}, B_{1}(s)=2(1-s) s, B_{2}(s)=s^{2},
$$

where $s$ stands for either of $\xi$ and $\eta$.

\section{Problem Solution}

In this section, first (in subsection 3.1) we introduce the necessary conditions which should be fulfilled to ensure the accurate representation of the spherical cap. Also, in subsection 3.2 we mathematically prove the incapability of the quadratic polynomials to perform this task.

Numerical and symbolic computations were performed using the commercial software MATLAB $^{\circledR}$.

\subsection{Necessary conditions}

The unknown position of the central control point $P_{11}\left(x_{11}, y_{11}, z_{11}\right)$, shown in Fig. 2, and the corresponding weight $w_{11}$ may be determined from the requirement that any point on the curvilinear patch of the unit sphere is desired to fulfill the condition:

$$
X^{2}+Y^{2}+Z^{2}=1
$$

Due to the symmetry of the spherical cap, we can immediately conclude that: 


$$
x_{11}=y_{11}=0
$$

Therefore, we only seek for the two variables $z_{11}$ and $w_{11}$.

Substituting Eqs. (5) to (7) into the key point Eq. (8), after elaboration it was found that the necessary condition $X^{2}+Y^{2}+Z^{2}=1$ becomes equivalent to the condition $F(\xi, \eta)=0$, where $F(\xi, \eta)$ is the following polynomial in two variables:

$$
\begin{aligned}
F(\xi, \eta) & =\left(a_{1} \xi \eta+a_{2} \xi \eta^{2}+a_{3} \xi \eta^{3}+a_{4} \xi \eta^{4}\right) \\
& +\left(a_{5} \xi^{2} \eta+a_{6} \xi^{2} \eta^{2}+a_{7} \xi^{2} \eta^{3}+a_{8} \xi^{2} \eta^{4}\right) \\
& +\left(a_{9} \xi^{3} \eta+a_{10} \xi^{3} \eta^{2}+a_{11} \xi^{3} \eta^{3}+a_{12} \xi^{3} \eta^{4}\right) \\
& +\left(a_{13} \xi^{4} \eta+a_{14} \xi^{4} \eta^{2}+a_{15} \xi^{4} \eta^{3}+a_{16} \xi^{4} \eta^{4}\right)
\end{aligned}
$$

with

$$
\begin{aligned}
& a_{1}=-\frac{4}{3}\left(6 w_{11}-2 \sqrt{3}\left(w_{11} z_{11}\right)+1\right) \\
& a_{2}=\frac{4}{3}\left[(18-4 \sqrt{6}) w_{11}+6(\sqrt{2}-\sqrt{3})\left(w_{11} z_{11}\right)+(3-2 \sqrt{6})\right] \\
& a_{3}=-\frac{16}{3}\left[2(3-\sqrt{6}) w_{11}+(3 \sqrt{2}-2 \sqrt{3})\left(w_{11} z_{11}\right)+(1-\sqrt{6})\right] \\
& a_{4}=\frac{8}{3}\left[2(3-\sqrt{6}) w_{11}+(3 \sqrt{2}-2 \sqrt{3})\left(w_{11} z_{11}\right)+(1-\sqrt{6})\right] \\
& a_{5}=\frac{4}{3}\left[(18-4 \sqrt{6}) w_{11}+6(\sqrt{2}-\sqrt{3})\left(w_{11} z_{11}\right)+(3-2 \sqrt{6})\right] \\
& a_{6}=-4\left[(18-8 \sqrt{6}) w_{11}+4 w_{11}{ }^{2}+(12 \sqrt{2}-6 \sqrt{3})\left(w_{11} z_{11}\right)\right. \\
& \left.-4\left(w_{11} z_{11}\right)^{2}+(7-4 \sqrt{6})\right] \\
& a_{7}=[96-(160 \sqrt{6}) / 3] w_{11}+32 w_{11}^{2}+(80 \sqrt{2}-32 \sqrt{3}) w_{11} z_{11} \\
& -32 w_{11}{ }^{2} z_{11}{ }^{2}+[48(\sqrt{6}+1)-(224 \sqrt{6}) / 3] \\
& a_{8}=16 w_{11}^{2} z_{11}^{2}-48 w_{11}+\left(80 \sqrt{6} w_{11}\right) / 3+(40 \sqrt{6}) / 3-16 w_{11}^{2}-40 \sqrt{2} w_{11} z_{11}+16 \sqrt{3} w_{11} z_{11}-24 \\
& a_{9}=-\frac{16}{3}\left[2(3-\sqrt{6}) w_{11}+(3 \sqrt{2}-2 \sqrt{3})\left(w_{11} z_{11}\right)+(1-\sqrt{6})\right] \\
& a_{10}=+32 w_{11}^{2}+\left[96-\frac{1}{3}(160 \sqrt{6})\right] w_{11}-32 w_{11}^{2} z_{11}^{2}+(80 \sqrt{2}-32 \sqrt{3}) w_{11} z_{11}+\left[48+48 \sqrt{6}-\frac{1}{3}(224 \sqrt{6})\right] \\
& a_{11}=-64 w_{11}^{2}+128\left(-1+\frac{2}{3} \sqrt{6}\right) w_{11}+64 w_{11}^{2} z_{11}^{2}+128\left(-\sqrt{2}+\frac{1}{3} \sqrt{3}\right) w_{11} z_{11}+\frac{128}{3}(-2+\sqrt{6}) \\
& a_{12}=32 w_{11}^{2}+[64-(128 \sqrt{6}) / 3] w_{11}-32 w_{11}^{2} z_{11}^{2}+[64 \sqrt{2}-(64 \sqrt{3}) / 3] w_{11} z_{11}+[128 / 3-(64 \sqrt{6}) / 3] \\
& a_{13}=\frac{8}{3}\left[2(3-\sqrt{6}) w_{11}+(3 \sqrt{2}-2 \sqrt{3})\left(w_{11} z_{11}\right)+(1-\sqrt{6})\right] \\
& a_{14}=-16 w_{11}^{2}+\left(-48+\frac{80}{3} \sqrt{6}\right) w_{11}+16 w_{111}^{2} z_{11}^{2}+(-40 \sqrt{2}+16 \sqrt{3}) w_{11} z_{11}+\left(-24+\frac{40}{3} \sqrt{6}\right) \\
& a_{15}=+32 w_{11}^{2}+[64-(128 \sqrt{6}) / 3] w_{11}-32 w_{11}^{2} z_{11}^{2}+64\left(\sqrt{2}-\frac{\sqrt{3}}{3}\right) w_{11} z_{11}+\frac{1}{3}(128-64 \sqrt{6}) \\
& a_{16}=-16 w_{11}^{2}+\left[-32+\frac{\mathrm{s}}{3} \sqrt{6}\right] w_{11}+16 w_{11}^{2} z_{11}^{2}+\left[-32 \sqrt{2}+\left(\frac{22}{3} \sqrt{3}\right)\right] w_{11} z_{11}+\frac{1}{3}(32 \sqrt{6}-64)
\end{aligned}
$$

If it was possible that equation (8) is fulfilled at any parametric point $P(\xi, \eta)$, then the polynomial $F(\xi, \eta)$ had to be identically equal to zero, thus any of its coefficients $\left(a_{i}, i=1, \ldots, 16\right)$ had to vanish:

$$
a_{i}=0, \quad i=1, \ldots, 16
$$

Within this context, below we shall investigate whether the sixteen conditions involved in Eq. (11) may be true together.
A close inspection on the separate coefficients involved in Eqs. (11) reveals that:

- The coefficients $a_{1}$ to $a_{5}$, as well as $a_{9}$ and $a_{13}$ are linear combinations of the variables $x_{1}=w_{11}$ and $x_{2}=w_{11} z_{11}$.

- We may observe that: $\left(a_{2}=a_{5}\right)$.

- The coefficient $a_{3}$ is identical to $a_{9}\left(a_{3}=a_{9}\right)$. Similarly, $a_{4}$ is identical to $a_{13} \quad\left(a_{4}=a_{13}\right)$. Also, $a_{3}=-2 a_{4}$. Therefore, only one of them four (i.e. $a_{3}, a_{4}, a_{9}$, and $a_{13}$ ), say $a_{3}=0$, may be considered as an independent necessary condition.

- Therefore, among the seven involved linear equations in (11), only the following three of them, i.e. $\left(a_{1}=0, a_{2}=0, a_{3}=0\right)$, are linearly independent. It is clarified that the therein involved variables are $x_{1}=w_{11}$ and $x_{2}=w_{11} z_{11}$.

- Regarding the nonlinear equations, we can notice that $\left(a_{12}=a_{15}=-2 a_{16}\right), a_{7}=a_{10}$ and $a_{8}=a_{14}$.

- Therefore, among the nine existing nonlinear nonlinear equations in (11), only five coefficients $\left(a_{6}=0, a_{7}=0, a_{8}=0, a_{11}=0, a_{12}=0\right) \quad$ are independent.

- Summarizing the above observations, the coefficients in monomials of the form $\xi^{i} \eta^{j}$ and $\xi^{j} \eta^{i}$ are equal each other.

\subsection{Findings on the solution of equations system}

In subsection 3.1 we found that there are three different equations systems: i.e., $\left(a_{1}=0, a_{2}=0\right)$, $\left(a_{2}=0, a_{3}=0\right)$ and $\left(a_{3}=0, a_{1}=0\right)$, which can be easily solved to determine the unknowns $x_{1}=w_{11}$ and $x_{2}=w_{11} z_{11}$. Each of these three cases is studied below.

\subsubsection{Case I: $\left(a_{1}=0, a_{2}=0\right)$}

Considering the first two equations of (11), i.e. $a_{1}=a_{2}=0$, we form a well-posed linear system in $w_{11}$ and $\left(w_{11} z_{11}\right)$, which eventually gives the following unique solution:

$$
w_{11}=\frac{1}{2} \quad \text { and } \quad z_{11}=\frac{4 \sqrt{3}}{3} \cong 2.3094011
$$




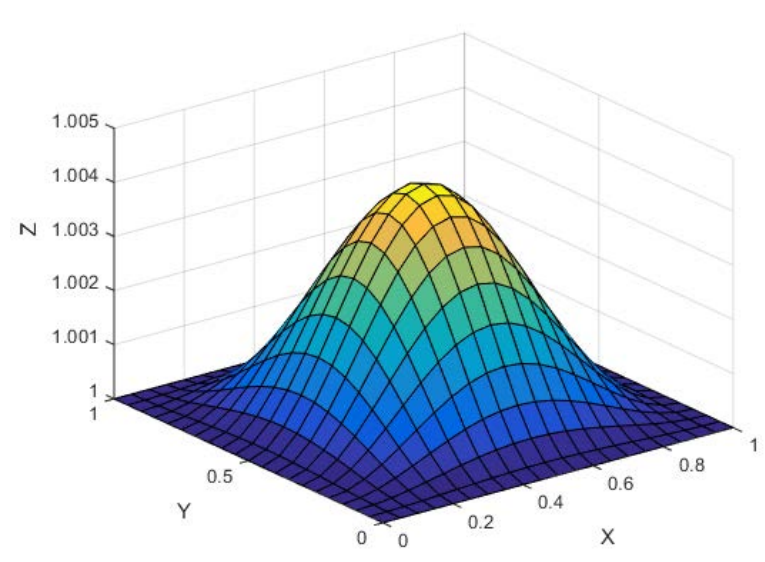

Fig. 3: Variation of computed radius.

Then, substituting Eq. (13) into Eq. (11), one obtains that five more terms vanish:

$$
a_{3}=a_{4}=a_{5}=a_{9}=a_{13}=0,
$$

while there are also nine non-vanishing terms as follows:

$$
\begin{aligned}
& a_{6}=1.3333, \quad a_{7}=-2.6667, a_{8}=1.3333, \\
& a_{10}=-2.6667, a_{11}=5.3333, \quad a_{12}=-2.6667, \\
& a_{14}=1.3333, \quad a_{15}=-2.6667, a_{16}=1.3333
\end{aligned}
$$

In other words, since the unique solution (13) fails vanishing the nine coefficients in Eq. (15), it turns out that there is no pair $\left(w_{11}, z_{11}\right)$ such as all the sixteen necessary conditions $a_{i}=0$ are fulfilled inside the spherical cap. Actually, the computed radius based on Eq. (13) varies in the interval $[1,1.0042]$ as shown in Fig. 3.

\subsubsection{Case II: $\left(a_{2}=0, a_{3}=0\right)$}

Considering the second and the third equations of (11), i.e. $a_{2}=a_{3}=0$, we obtain again the solution shown in Eq. (13).

\subsubsection{Case III: $\left(a_{3}=0, a_{1}=0\right)$}

Considering the first and the third equations of (11), i.e. $a_{1}=a_{3}=0$, we obtain again the solution shown in Eq. (13).

\subsubsection{Least-squares}

Finally, all the first three equations of (11) were considered to occur simultaneously as a set. The well-known least squares leads again to the same solution given by Eq. (13).

\section{An approximate optimal solution}

In this section we present a practical way to minimize the average deviation between the computed and the desired accurate (ideally spherical) patch.

Since the direct mathematical procedure of section 3 fails to determine a unique solution that accurately fulfills the constraint of Eq. (8), we resort to a different procedure that will satisfy it in an approximate manner.

Within this context, to minimize the overall error in the approximation of the spherical cap, we seek for a numerical solution based on non-linear mathematical optimization techniques, which of course will not fulfill all the conditions $a_{i}, i=1, \ldots, 16$.

One of the efficient methods is the second order Newton's method, and particularly the Sequential Quadratic Programming (SQP), which was

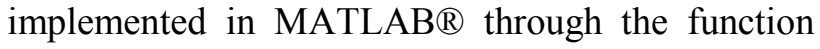
fmincon [24]. The formulation is specified as follows:

Find the minimum of the function $f(\mathbf{x})$, which here comprises two variables $\mathbf{x}=\left[x_{1}, x_{2}\right]^{T}$ under the following constraints:

$$
\left.\begin{array}{l}
c(\mathbf{x}) \leq 0 \\
c_{e q}(\mathbf{x})=0 \\
\mathbf{A} \cdot \mathbf{x} \leq \mathbf{b} \\
\mathbf{A}_{e q} \cdot \mathbf{x} \leq \mathbf{b}_{e q} \\
l_{b} \leq \mathbf{x} \leq u_{b}
\end{array}\right\}
$$

where $\mathbf{b}$ and $\mathbf{b}_{e q}$ are vectors, $\mathbf{A}$ and $\mathbf{A}_{e q}$ are matrices, $c(\mathbf{x})$ and $c_{e q}(\mathbf{x})$ are constraint functions that return vectors, and $f(\mathbf{x}), \mathbf{x}$, as well as the lower and upper bounds $l_{b}$, and $u_{b}$ can be passed as vectors or matrices.

The objective function $f(\mathbf{x})$ to be minimized is the sum of the squares of the deviations between the calculated (using Eq. (5)) and the accurate radius $R$ of the spherical cap at $n$ discrete pairs of parameters $(\xi, \eta)$ :

$$
f\left(w_{11}, z_{11}\right)=\sum_{k=1}^{n}\left[\left(\sum_{i=0}^{2} \sum_{j=0}^{2} R_{i j}(\xi, \eta) x_{P_{i j}}\right)^{2}+\left(\sum_{i=0}^{2} \sum_{j=0}^{2} R_{i j}(\xi, \eta) y_{P_{i j}}\right)^{2}+\left(\sum_{i=0}^{2} \sum_{j=0}^{2} R_{i j}(\xi, \eta) z_{P_{i j}}\right)^{2}-R^{2}\right]^{2}
$$




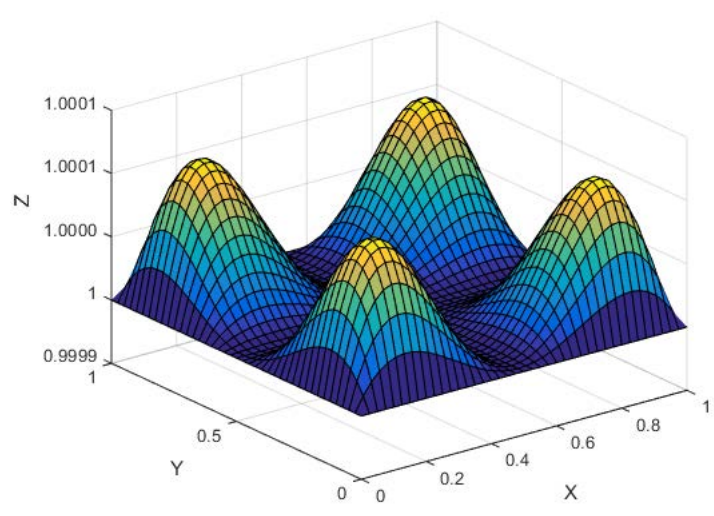

Fig. 4: Optimal variation of the calculated radius

The most necessary position that should be included in the $k$-sum of Eq. (17) is the central point at $(\xi=\eta=0.5)$, but also more characteristic points may be taken into account.

Moreover, equality constraints $c_{e q}(\mathbf{x})$ may be imposed for the accurate approximation of the patch at particular pairs $(\xi, \eta)$.

In general, both the particular chosen objective function and the equality constraints affect the optimal solution to some extent. Between several choices, one of them (perhaps the optimal) gives:

$$
\left.\begin{array}{l}
w_{11}=0.467959158138040 \\
z_{11}=2.370695072453977
\end{array}\right\},
$$

which leads to calculated radii $R_{\text {calculated }}$ that are found within the interval:

$$
R_{\text {calculated }} \in[0.999999994,1.000104146],
$$

while the corresponding variation of calculated radius is shown in Fig. 4 and is the same for all the four quadrants in which the unit square may be divided.

\section{Discussion and future work}

It is well known that when performing knot insertion or degree elevation to a tensor product NURBS surface, the shape is parametrically preserved [23]. In this framework, the findings of this paper suggest that if isogeometric analysis (IGA) is to be performed to a spherical cap, we should not start it with a quadratic representation of the geometry, because thus we would had to deal with an inaccurate geometry, which would continue to be so after the knot insertion or degree elevation. This finding is in contrast to previous experience, where a circular arc could be initially idealized by three control points using rational quadratic Bernstein polynomials, and then knot insertion (or degree elevation) would follow.

So far, it is established that a NURBS patch of fourth degree is capable of accurately representing a spherical cap (see, $[16,17]$ ), thus we take for granted that isogeometric analysis should start from this configuration. A future research should focus on the capability of rational cubic representation of the spherical cap.

\section{Conclusion}

It was shown that quadratic rational approximation is not adequate to accurately representing a spherical cap. The analytical equation of the sphere led to a system of seven linear and nine nonlinear (quadratic) equations, which unfortunately do not give a unique solution. A least-squares procedure in conjunction with the sequential quadratic programming method has given a practically acceptable solution for the position of the central control point and its associated weight, which leads to computed radii in the parametric space within the interval [0.999999994, 1.000104146].

\section{Appendix A}

\section{Control points of the circular arc $A B$ in the spherical cap}

Here we shall study the control points and the corresponding weights associated to the circular arc

$A B$, on a great circle of a sphere $(O, R)$.

The coordinates of the ends $A$ and $B$ of the above-mentioned circular arc are given by Eq. (3). The control point $P_{00}$ coincides with $A$ whereas $P_{02}$ coincides with $B$ (see, Fig. 2). Moreover, according to [15] the intermediate control point $P_{01}$ is found at the intersection of the tangent lines of the circular arc $A B$ at points $A$ and $B$ on the plane $O A B$.

Let $M$ be the middle of the straight segment (chord) $A B$, as shown in Fig. 5. Obviously we have:

$$
\overrightarrow{O M}=\frac{\overrightarrow{O A}+\overrightarrow{O B}}{2}=0 \vec{i}-\frac{a}{2} \vec{j}+\frac{a}{2} \vec{k},
$$

where $(\vec{i}, \vec{j}, \vec{k})$ is the triplet of the unit vectors on the three Cartesian axes $(x, y, z)$, respectively. Therefore, the length of the segment $O M$ is

$$
|\overrightarrow{O M}|=\sqrt{0^{2}+\left(-\frac{a}{2}\right)^{2}+\left(\frac{a}{2}\right)^{2}}=\frac{a}{\sqrt{2}}
$$


Obviously, the line $O P_{01}$ passes through $M$ and is perpendicular to $A B$. The application of Pythagorean theorem on the rectangular triangle $O M B$ at $M$, $(O M)^{2}+(B M)^{2}=(O B)^{2}$, in conjunction with $(B M) \square(A B) / 2$ and $(A B) \square a$, yields:

$$
\left(\frac{a}{\sqrt{2}}\right)^{2}+\left(\frac{a}{2}\right)^{2}=R^{2},
$$

from which Eq. (3) is deduced.

Let now $\alpha$ be the central angle $\square(O A, O B)$, which is double than the equal angles $\square\left(O P_{11}, O B\right)=\square\left(B A, O P_{11}\right)=\alpha / 2$.

Since

$O B=O P_{11} \cos (\alpha / 2)$, and $O B=R$, we get

$$
O P_{11}=\frac{R}{\cos (\alpha / 2)}
$$

According to [15], we have that the weight associated to the control point $P_{01}$ should be given by

$$
w_{01}=\cos \frac{\alpha}{2}
$$

Considering the definition of the cosine by

$$
\cos \frac{\alpha}{2} \square \frac{(O M)}{(O B)}
$$

and substituting $(O M)$ by (A-2) while $(O B)=R$, Eq. (A-6) becomes:

$$
\cos \frac{\alpha}{2}=\sqrt{\frac{2}{3}}
$$

Therefore, combining Eq. (A-5) with (A-7), the weight associated to the control point $P_{11}$ should be

$$
w_{01}=\sqrt{\frac{2}{3}}
$$

Regarding the position of the control point $P_{11}$, we have:

$$
\overrightarrow{O P}_{11}=\left|\overrightarrow{O P}_{11}\right| \cdot \frac{\overrightarrow{O M}}{|\overrightarrow{O M}|}=\overrightarrow{O M} \cdot \frac{\left|\overrightarrow{O P}_{11}\right|}{|\overrightarrow{O M}|}
$$

Substituting $\overrightarrow{O M}$ by (A-1), $\left|\overrightarrow{O P}_{11}\right|$ by (A-4) and (A-7), while $|\overrightarrow{O M}|$ by (A-2), Eq. (A-9) eventually leads to:

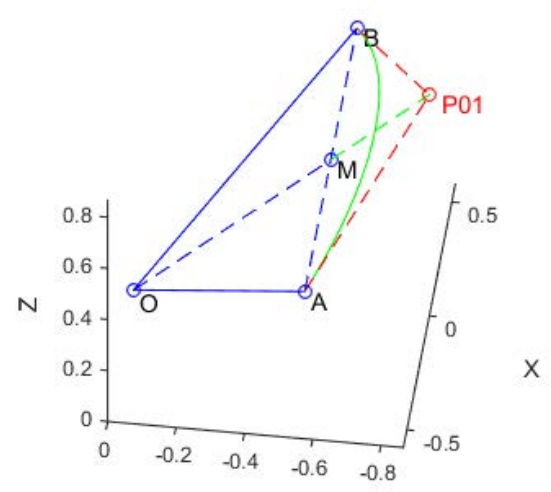

Y

Fig. 5: Construction for the control points

$$
\overrightarrow{O P}_{11}=0 \vec{i}-\frac{3 a}{4} \vec{j}+\frac{3 a}{4} \vec{k}
$$

\section{References:}

[1] Farin G., Hoschek J., and Kim M-S (Eds.), Handbook of Computer Aided Geometric Design, North Holland, 2002.

[2] Zienkiewicz O.C., Taylor R.L, Zhu J.Z., The Finite Element Method: Its Basis and Fundamentals, 7th Edition, ButterworthHeinemann (September 5, 2013).

[3] Hughes T.J.R., Cottrell J.A., Bazilevs Y., Isogeometric analysis: $\mathrm{CAD}$, finite elements, NURBS, exact geometry and mesh refinement, Computer Methods in Applied Mechanics and Engineering, Vol. 194 (39-41), 1 October 2005, pp. 4135-4195.

[4] Cottrell J.A., Hughes T.J.R., Bazilevs Y., Isogeometric Analysis: Toward Integration of $C A D$ and FEA, John Wiley \& Sons, 2009.

[5] Rypl D., Patzák B., From the finite element analysis to the isogeometric analysis in an object oriented computing environment, Advances in Engineering Software, 44(2012) 116-125.

[6] Dupuy J., Heitz E., and Belcour L., A Spherical Cap Preserving Parameterization for Spherical Distributions, ACM Transactions on Graphics, Vol. 36, No. 4, Article 139. Publication date: July 2017.

[7] Etayo, U. Spherical Cap Discrepancy of the Diamond Ensemble. Discrete Comput Geom (2021). https://doi.org/10.1007/s00454-02100305-4

[8] Song Jia-Yong, Zeng Rui-Min, Xu Dao-Yun, Wang Yi, Ding Zhao, Yang Chen, A compact AAA-compatible multispectral solar simulator 
based on spherical cap chamber, Solar Energy, Vol. 220, 15 May 2021, Pages 1053-1064.

[9] Thanh L.T., Minh L.H., Doumbia V., AmoryMazaudier C., Dung N.T., and Chau Ha Duyen, A spherical cap model of the geomagnetic Beld over southeast Asia from CHAMP and Swarm satellite observations, J. Earth Syst. Sci. (2021) 130:13 (https://doi.org/10.1007/s12040-020$\underline{01507-9)}$

[10] Abdulkadir M., Ugwoke B., Abdulkareem L.A., Zhao D., and Hernandez-Perez V., Experimental investigation of the characteristics of the transition from spherical cap bubble to slug flow in a vertical pipe, Experimental Thermal and Fluid Science, Volume 124, 1 June 2021, 110349.

[11] Gee M., Wall W.A., and Ramm E. (2005). Parallel multilevel solutions of nonlinear shell structures, Computer Methods in Applied Mechanics and Engineering, 194:2513-2533.

[12] Stanley, G.M. (1985). Continuum-based Shell Elements. $\mathrm{PhD}$ thesis, Division of Applied Mechanics, Stanford University.

[13] Sankar A., Natarajan S., Merzouki T., and Ganapathi M., Nonlinear Dynamic Thermal Buckling of Sandwich Spherical and Conical Shells with CNT Reinforced Facesheets, International Journal of Structural Stability and Dynamics, Vol. 17, No. 9 (2017) 1750100 (20 pages).

[14] Hajlaoui A., Triki E., Frikha A., Wali M., Dammak F., Nonlinear Dynamics Analysis of FGM Shell Structures with a Higher Order Shear Strain Enhanced Solid-Shell Element, Latin American Journal of Solids and $\begin{array}{llll}\text { Structures } & 14 & \text { (2017) } & 72-91\end{array}$ (http://dx.doi.org/10.1590/1679-78253323)

[15] Piegl L., and Tiller W., A Menagerie of Rational B-Spline Circles, IEEE Computer Graphics and Applications, vol. 9, September/October 1989, pp. 48-56, DOI Bookmark: 10.1109/38.35537

[16] Cobb J.E., Tiling the Sphere with Rational Bézier Patches, TR UUCS-88-009, 1988. Download from: https://core.ac.uk/download/pdf/276277507.pdf

[17] Cobb J.E., Letter to the editor, Computer Aided Geometric Design, Vol.6, No.1, pp. 85-86.

[18] Carl de Boor, Spline Toolbox for Use with MATLAB ${ }^{\circledR}$, Users Guide, Version 3, The MathWorks Inc., 2000, p. 1-30.

[19] Boehm W., and Hansford D., Parametric representation of quadric surfaces, RAIRO Modélisation Mathématique et Analyse Numérique, Vol.26, No.1, 1992, pp. 191-200.
[20] Hansford D., Barnhill R.E., Farin G., Curves with quadric boundary precision, Computer Aided Geometric Design, 11 (1994), 519-531.

[21] Amer R.B.M., Abd El- Mageed M.A., Design Quadratic Patch and Cubic Patch of the Surface, IOSR Journal of Mathematics (IOSRJM), e-ISSN: 2278-5728. Volume 5, Issue 2 (Jan. - Feb. 2013), PP 23-33.

[22] Lausegger M., Konstruktionen rationaler Flaechenstücke auf Quadriken und deren geometrische eigenschaften, Diplomarbeit, Technische Universität Wien, Institut für Diskrete Mathematik und Geometrie, 2015 (supervised by Prof. Martin Peternell).

[23] Piegl L., Tiller W., The NURBS Book, Springer, 1995.

[24] Hock W., Schittkowski K., A Comparative Performance Evaluation of 27 Nonlinear Programming Codes, Computing, Vol.30, 1983, p. 335-358.

[25] Provatidis C.G, Precursors of Isogeometric Analysis: Finite Elements, Boundary Elements and Collocation Methods, Springer, Cham, 2019.

Contribution of Individual Authors to the
Creation of a Scientific Article (Ghostwriting
Policy)
The author contributed in the present research, at all The author contributed in the present research, at all
stages from the formulation of the problem to the final findings and solution.

\section{Sources of Funding for Research Presented in a Scientific Article or Scientific Article Itself}

No funding was received for conducting this study.

\section{Conflict of Interest}

The author has no conflict of interest to declare that is relevant to the content of this article.

Creative Commons Attribution License 4.0 (Attribution 4.0 International, CC BY 4.0)

This article is published under the terms of the Creative Commons Attribution License 4.0 https://creativecommons.org/licenses/by/4.0/deed.en US 